\title{
THE EC14026 STARS - PULSATING HOT SUBDWARFS
}

\author{
C. KOEN, D. O'DONOGHUE, D. KILKENNY AND R.S. STOBIE \\ South African Astronomical Observatory, PO Box 9, Observatory \\ 7935, Cape, South Africa
}

\section{Introduction}

The Edinburgh-Cape Blue Object Survey (Stobie et al. 1997a) is a southern hemisphere survey to discover hot, blue stellar objects brighter than $\mathrm{B}=18$ at galactic latitudes more than $30^{\circ}$ from the galactic plane. The main categories of object detected are hot subdwarfs, white dwarfs, blue horizontal branch stars, apparently normal B stars, cataclysmic variables and (stellar-like) active galactic nuclei. Over $50 \%$ of the EC Survey comprises hot subdwarfs.

In the course of high speed photometry runs on selected EC objects (e.g. candidate pulsating white dwarfs) a whole new class of rapidly pulsating stars was discovered serendipitously. These stars are now recognised to be pulsating sdB stars, dubbed the EC14026 stars after the prototype. Since the discovery of the pulsations and the realisation that this was a new class of rapid pulsators, a major search was initiated for other pulsators. Over $300 \mathrm{sdB}$ stars have now been searched using the techniques of high speed photometry and frequency analysis, and to date (July 1997) twelve pulsators have been discovered.

This paper will summarise the results of the first four pulsators (Kilkenny et al. 1997 - paper I; Koen et al. 1997 - paper II; Stobie et al. 1997b - paper III; O'Donoghue et al. 1997a - paper IV), present photometry on two new pulsators, and preliminary results on three other pulsators.

\section{Photometric Analysis}

To discover new pulsators high speed photometry in white light with a time resolution of $10 \mathrm{~s}$ is obtained for 1-2 hours. Both photoelectric photometers and a high time-resolution CCD camera have been used with $0.5-\mathrm{m}$ to $1-\mathrm{m}$ class telescopes. The data are analysed for coherent frequencies by periodogram techniques. For stars with $\mathrm{B}<15$ it is normally possible to detect pulsations with semi-amplitude $>3$ mmag. Once pulsations are confirmed the star is 


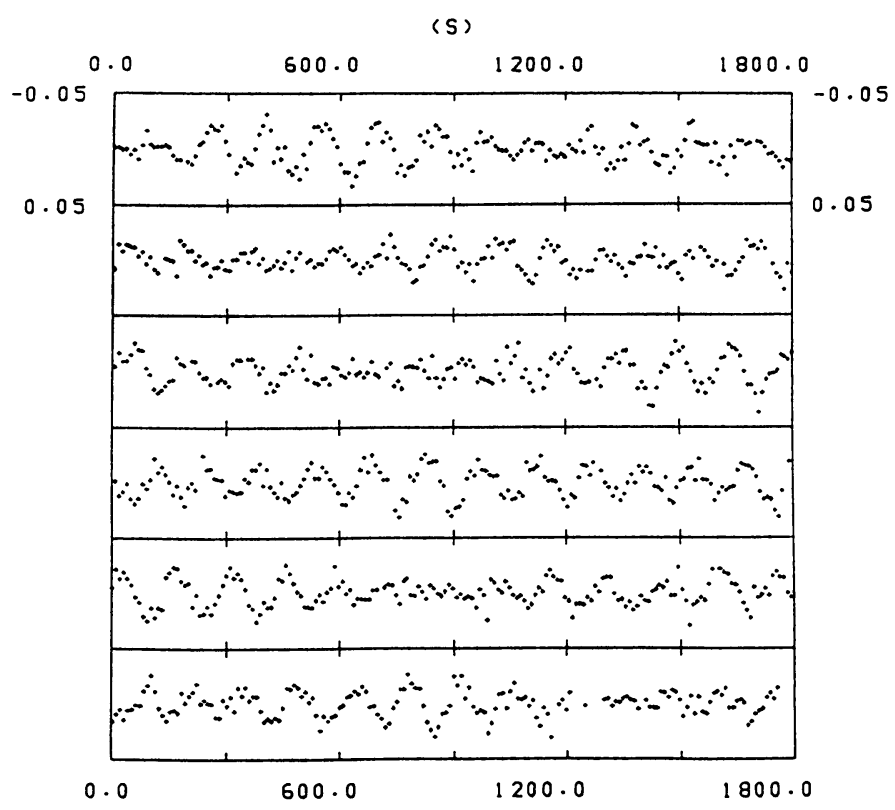

Figure 1. Light curve of pulsating sdB star PG1047+003. The ordinate units are differential magnitudes.

followed intensively for a number of nights. The details of the photometric techniques are described in papers I-IV.

Fig. 1 shows the time series photometry of a new pulsator PG1047+003, (O'Donoghue et al. 1998). Oscillations with a period of $\sim 140 \mathrm{~s}$ and a peak-topeak amplitude of several hundredths of a magnitude are evident. The multiperiodic nature of the oscillations is evident from the 'beating' phenomenon. The periodogram of this photometric run is shown in Fig. 2 revealing five frequencies well above the noise (the peak at $120 \mathrm{~s}$ is spurious, caused by the drive error of the $0.75-\mathrm{m}$ telescope). Frequency analysis of all the nights reveals at least 9 periods in the range 104-162 s.

Other EC14026 stars have similar pulsation properties, though mostly not with such a large peak-to-peak amplitude. In Table 1 we list the properties of eight of the twelve pulsators that have been studied to date. The three dominant pulsation periods of each pulsator are listed together with their semiamplitudes. Note that many of these semi-amplitudes are variable in time, possibly as a result of beating between close frequencies. In some cases (PB8783, PG1047+003) many more than three periods are known. The EC14026 stars listed are observed to be multiperiodic with periods in the range $90 \mathrm{~s}<\mathrm{P}<$ $220 \mathrm{~s}$ and semi-amplitudes of component frequencies normally $<10 \mathrm{mmag}$. These pulsating hot subdwarfs are amongst the most rapid pulsators known, comparable to the shortest-period white dwarf pulsators.

High speed photometry of the largest amplitude pulsator, PG1605+072, (not listed in Table 1) is illustrated in Fig. 3. This pulsator is unusual in 


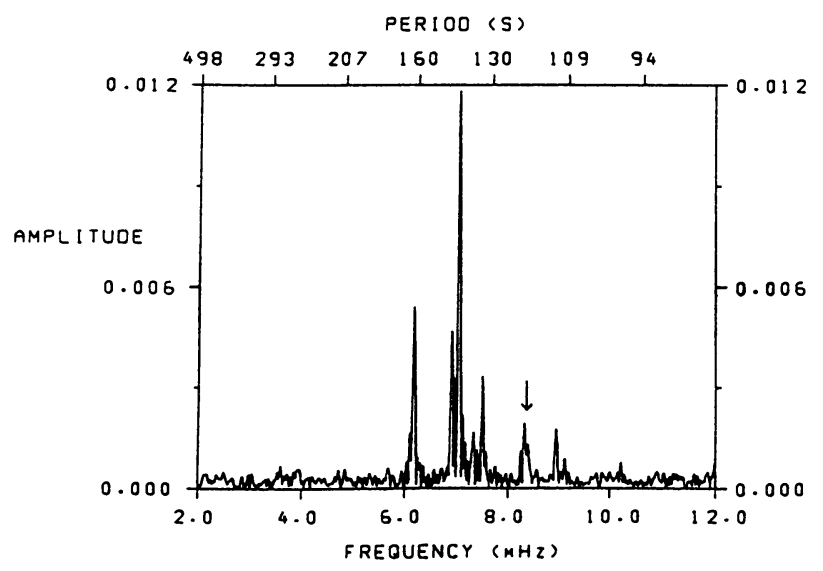

Figure 2. Fourier amplitude spectrum of $5.7 \mathrm{~h}$ high speed photometry of PG1047+003. The arrow indicates the frequency of the SAAO $0.75-\mathrm{m}$ telescope drive.

comparison to the properties of the other pulsators because of its much larger amplitude $\quad(\sim 0.3$ mag peak-to-peak $)$, the length of the periods of oscillation

TABLE 1. Spectroscopic and pulsation properties of EC14026 stars

\begin{tabular}{lccccccccc}
\hline star & $\begin{array}{c}\text { spectral } \\
\text { type }\end{array}$ & $\mathrm{T}_{\text {eff }}$ & $\log \mathrm{g}$ & $\begin{array}{c}\mathrm{P}_{1} \\
(\mathrm{~s})\end{array}$ & $\begin{array}{c}\mathrm{a}_{1} \\
\mathrm{mmag}\end{array}$ & $\begin{array}{c}\mathrm{P}_{2} \\
(\mathrm{~s})\end{array}$ & $\begin{array}{c}\mathrm{a}_{2} \\
\mathrm{mmag}\end{array}$ & $\begin{array}{c}\mathrm{P}_{3} \\
(\mathrm{~s})\end{array}$ & $\begin{array}{c}\mathrm{a}_{3} \\
\mathrm{mmag}\end{array}$ \\
\hline EC14026-2647 & sdB+G2V & 34700 & 6.1 & 144 & 12 & 134 & 4 & - & - \\
PB8783 & sdB+F3V & 35700 & 5.6 & 122 & 9 & 123 & 4 & 134 & 2 \\
EC10228-0905 & sdB+G0V & 35100 & 6.0 & 140 & 7 & 152 & 5 & 147 & 4 \\
EC20117-4014 & sdB+F6V & 34800 & 5.9 & 137 & 4 & 142 & 1 & 157 & 1 \\
PG1047+003 & sdB & 35000 & 5.9 & 142 & 10 & 162 & 5 & 145 & 4 \\
PG1336-018 & sdB+M5V & 33000 & 5.7 & 184 & 10 & 141 & 5 & - & - \\
EC05217-3914 & sdB & - & - & 217 & 5 & 214 & 3 & - & - \\
EC11583-2708 & sdB+ & - & - & 149 & 5 & 143 & 3 & - & - \\
\hline
\end{tabular}

$\sim 480 \mathrm{~s}$, and the large number of frequencies identifiable on a single night (at least 15). As this pulsator presents one of the best opportunities for mode identification, a multisite campaign was recently organised to maximise the number of frequencies identified and to minimise the alias problems. More than 30 frequencies have been identified in the preliminary analysis.

The potential of the pulsations of the EC14026 stars as diagnostic probes of their interiors can only be realized fully by intensive campaigns of multisite time series photometry. To this end a campaign spanning 2 weeks and involving data from 5 different sites was carried out on PB8783 (O'Donoghue et 


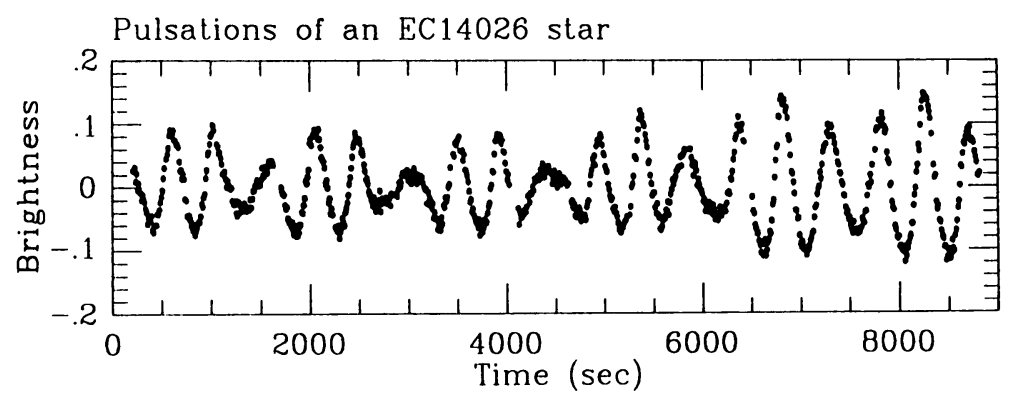

Figure 3. Light curve of largest amplitude sdB pulsator. The ordinate units are fractional changes in intensity.

al. 1997b - Paper V). Frequency analysis yielded at least 12 pulsation periods in 7 discrete groups between 94 and $136 \mathrm{~s}$. Three of the periods in one specific group are equally-spaced in frequency with a spacing of $0.96 \mu \mathrm{Hz}$, implying a rotation period of $\sim 12 \mathrm{~d}$. The periods of a detailed model of an evolved extreme horizontal branch sdB star give good agreement with the observed periods (table 3 of paper V). Although the period agreement is good, we caution against this being the only possible mode identification. The problem is that in pulsation models of sdB stars, the frequency spacing between modes with different $n$ and/or $\ell$ is occasionally very small (e.g. for $n=3, \ell=0,2$ in table 3 of paper V) - whereas most observed spacings are much larger. Although the exact values of $n$ and $\ell$ must await detailed modelling, small values are implied.

\section{Spectroscopic Analysis}

Low $(\sim 3.5 \AA)$ and medium $(\sim 1.2 \AA)$ resolution spectra have been obtained of the EC14026 pulsators using the SAAO 1.9-m telescope equipped with an intensified Reticon spectrograph. The spectra all show broad, strong Balmer lines superimposed on a blue continuum, characteristics of sdB stars. However, some spectra also show (unexpectedly for sdB stars) the CaII $\mathrm{K}$ line and sometimes the G-band. These features are indicative of a composite spectrum comprising a hot sdB star and a cool main sequence star. The details of the fitting technique to derive the best estimate of the spectral types of the two components and the values of $\mathrm{T}_{\text {eff }}$ and $\log \mathrm{g}$ for the $\mathrm{sdB}$ stars are given in paper IV and the results listed in Table 1.

Optical spectroscopy will only detect a main sequence companion to an $\mathrm{sdB}$ star if the companion is of spectral type earlier than $\sim \mathrm{G} 5$. For later $\mathrm{K}$ and $\mathrm{M}$ spectral types infrared photometry is required to detect the presence of a companion. In Table 1 the fraction of composite systems is 5 out of 8 . This is a minimum and it is conceivable that all EC14026 stars are binary in nature. 


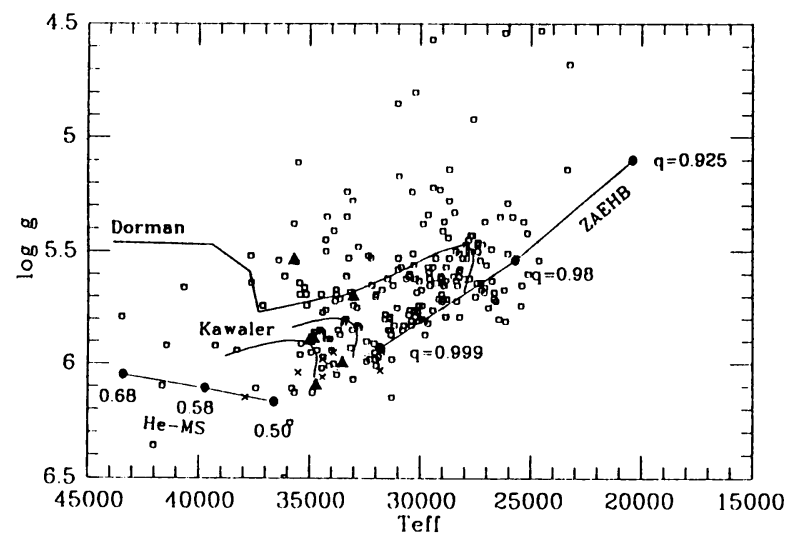

Figure 4. The $\log \mathrm{g}-\mathrm{T}_{\text {eff }}$ plane showing sdB field subdwarfs (open symbols) and EC14026 pulsators (filled triangles)

It should be noted that the M5 spectral type of the companion in PG1336-018 was estimated not from the spectroscopic analysis but by the reflection effect in this close binary with $\mathrm{P}_{\text {orb }}=0.1010174 \mathrm{~d}$ (Kilkenny et al. 1998).

The position of the sdB oscillators in the $\log \mathrm{g}-\mathrm{T}_{\text {eff }}$ plane is shown in Fig. 4 by the filled triangles. The open symbols are $\log g$ and $\mathrm{T}_{\text {eff }}$ determinations for a large sample of field sdB subdwarfs by Saffer et al. (1994). The zero age extreme horizontal branch, the helium main sequence and evolutionary tracks are shown. With the exception of PG1605+072, the range of $\log \mathrm{g}$ (5.6 to 6.1) and $\mathrm{T}_{\text {eff }}(33000$ to $35700 \mathrm{~K}$ ) exhibited by the EC14026 stars occupies a small fraction of the subdwarf parameter space, suggestive of an instability strip. The sdB stars lie on the extended blue horizontal branch and as such are core helium burning stars comprised of a $\sim 0.5 \mathrm{M}_{\odot}$ helium core surrounded by a thin $\left(\mathrm{M}_{\text {env }}<0.01 \mathrm{M}_{\odot}\right)$ hydrogen envelope. The pulsators at the hot end of the sdB star range contain even thinner $\left(\mathrm{M}_{\mathrm{env}}<0.0005 \mathrm{M}_{\odot}\right)$ hydrogen envelopes.

\section{Discussion}

Pulsation models by Kawaler (see paper III) with 'standard' zero-age horizontal branch (ZAHB) models have been constructed to compare the observed and theoretical pulsation periods. The models have a helium core mass of $0.485 \mathrm{M}_{\odot}$ with a hydrogen layer thickness of only $\sim 0.00015 \mathrm{M}_{\odot}$ to bring their temperatures into agreement with observation. The periods of the low order $(\ell=0,1,2 ; n=0,1,2) \mathrm{p}$-modes are in the observed range. It is not expected that higher order $\ell$ modes would be easily visible because of geometric cancellation effects. Furthermore, it is clear from the stars with three or more observed periods that not all the modes can be radial.

Although the periods of the models agree with the observed range, none of the above models was pulsationally unstable. However, independently of 
the observational discovery of these pulsators, Charpinet et al. (1996) demonstrated that significant driving in sdB stars could be caused by the opacity bump associated with heavy element ionization (similar to the instability mechanism in $\beta$ Cephei stars). This could lead to pulsational instability if the heavy element abundance in the relevant zone was high enough $(\mathrm{Z} \geq 0.04)$. In a later paper, Charpinet et al. (1997) presented improved models with nonuniform iron abundance distributions obtained through the condition of diffusive equilibrium between gravitational settling and radiative levitation. The enhanced iron abundance led to unstable models for low order radial and nonradial pulsation modes in the range $36500>\mathrm{T}_{\text {eff }}>29000 \mathrm{~K}$. These and other results are presented in more detail by Fontaine in this volume.

Once modes have been identified these stars represent an excellent laboratory for elucidating the structure of sdB stars. This could lead to a better understanding of how sdB stars evolve into this state from their red giant progenitors. Furthermore, with the short periods and ability to measure period change within a few years it should be possible to determine the evolutionary time scale of core helium burning on the horizontal branch.

\section{References}

Charpinet, S., Fontaine, G., Brassard, P. and Dorman, B. (1996) The potential of asteroseismology for hot, subdwarf B stars: A new class of pulsating stars?, Astrophys. J. 471, pp L103-106

Charpinet, S., Fontaine, G., Brassard, P., Chayer, P., Rogers, F.J., Iglesias, C.A. and Dorman, B. (1997) A Driving Mechanism for the Newly Discovered Class of Pulsating Subdwarf B Stars, Astrophys. J., in press

Kilkenny, D., Koen, C., O'Donoghue, D. and Stobie, R.S. (1997) A new class of rapidly pulsating star - I. EC14026-2647, the class prototype, Mon. Not. Roy. astr. Soc. 285, pp 640-644 (Paper I)

Kilkenny, D., O'Donoghue, D., Koen, C. and van Wyk, F. (1998) The EC14026 stars - VII. PG1336-018 - a pulsating sdB star in an HW Vir-type eclipsing binary, preprint (Paper VII)

Koen, C., Kilkenny, D., O'Donoghue, D., van Wyk, F. and Stobie, R.S. (1997) A new class of rapidly pulsating star - II. PB8783, Mon. Not. Roy. astr. Soc. 285, pp 645-650 (Paper II)

O’Donoghue, D., Lynas-Gray, A.E., Kilkenny D., Stobie, R.S. and Koen, C. (1997a) A new class of rapidly pulsating star - IV. Oscillations in EC20117-4014 and atmospheric analysis, Mon. Not. Roy. astr. Soc. 285, pp 657-672 (Paper IV)

O'Donoghue, D., Koen, C., Solheim, J.-E., Barstow, M.A., Dobbie, P.D., O'Brien, M.S., Clemens, J.C., Sullivan, D.J. and Kawaler, S.D. (1997b) The EC14026 stars -V. The pulsation periods of PB8783, preprint (Paper V)

O'Donoghue, D., Koen, C., Lynas-Gray, A.E., Kilkenny, D. and van Wyk, F. (1998) The EC14026 stars - VI. PG1047+003, preprint (Paper VI)

Saffer, R.A., Bergeron, J., Koester, D. and Liebert, J. (1994) Atmospheric parameters of field subdwarf B stars, Astrophys. J. 432, pp 351-366

Stobie, R.S. et al. (1997a) The Edinburgh-Cape Blue Object Survey - I. Description of the survey, Mon. Not. Roy. astr. Soc. 287, pp 848-866

Stobie, R.S., Kawaler, S.D., Kilkenny, D., O'Donoghue, D. and Koen, C. (1997b) A new class of rapidly pulsating star - III. Oscillations in EC10228-0905 and pulsation analysis, Mon. Not. Roy. astr. Soc. 285, pp 651-656 (Paper III) 